\title{
Cochlear Implants for Patients With Common Cavity Deformities and the Impact of Electrode Positioning
}

\author{
Seong Hoon Bae (iD · Jihoon Choi (iD · Jae Young Choi iD \\ Department of Otorhinolaryngology, Yonsei University College of Medicine, Seoul, Korea
}

Objectives. Common cavity deformity is a rare congenital bony labyrinth malformation associated with profound hearing loss. Cochlear implants are widely used for hearing rehabilitation for common cavity deformities; however, the reported prognosis is poor. Due to the deformed anatomical structure, it is important to consider the position of the electrodes to maximize the performance of the cochlear implant. The present study discusses the impact of electrode placement on hearing outcomes.

Methods. A retrospective medical chart review of eight common cavity deformity patients (10 cochlear implants) who received cochlear implants was performed at a single university hospital. In all eight patients, implant surgery was performed using single-slit labyrinthotomy. Electrodes wer e manually bent before insertion to prevent misplacement and to reduce physical damage to the neuroepithelium.

Results. Four of the 10 electrodes were misplaced, with their tips placed in the anterior semicircular canal or internal auditory canal. However, after implant surgery, all patients — including those with misplaced electrodes—gained auditory perception and improved hearing function. One patient who had electrodes that did not contact the inner wall of the cavity showed limited activity of the electrodes $(27 \%)$ compared to others $(64 \%-100 \%)$.

Conclusion. Proper contact of the electrode with the inner wall was more likely to be important for cochlear implant success in cases of common cavity deformity than appropriate placement of the electrode tip.

Keywords. Cochlear Implants; Sensorineural Hearing Loss; Congenital Abnormalities; Inner Ear

\section{INTRODUCTION}

Common cavity deformity is a rare congenital bony labyrinth malformation associated with profound hearing loss. The prevalence of common cavity deformity as a proportion of cochlear malformations is variable, ranging from $0.7 \%$ to $25 \%$ [1]. A single cystic cochlear-vestibular structure is a representative feature of common cavity deformity, but it also has wide phenotypic variability in the internal auditory canal (IAC), semicircular canals, and cochlear nerve. In recent years, many groups have treat-

\footnotetext{
- Received April 15, 2021

Revised May 31, 2021

Accepted June 10, 2021

- Corresponding author: JaeYoung Choi

Department of Otorhinolaryngology, Severance Hospital, Yonsei University

Health System, Yonsei University College of Medicine, 50-1 Yonsei-ro,

Seodaemun-gu, Seoul 03722, Korea

Tel: +82-2-2228-3484, Fax: +82-2-393-0580

E-mail: jychoi@yuhs.ac
}

ed common cavity deformity patients using cochlear implants. However, the prognosis of patients with common cavity deformity after receiving cochlear implants is not encouraging when compared with patients with other cochlear deformations [2-4].

The biggest challenge in performing cochlear implant surgery for common cavity deformity is the distorted anatomical structure. Both the bony labyrinth structure and the spiral ganglion, the target of electric stimulation, are absent. However, an abundance of evidence regarding hearing improvements after cochlear implant surgery implies that a remnant of the spiral ganglion is also present in common cavity deformities [2-5]. After Miyamoto et al. [6] first performed a cochlear implant for common cavity deformity in 1986, several techniques for electrode positioning in the cavity have been suggested. Most techniques are focused on the stability of electrode positioning near the IAC fundus, where the remnant spiral ganglion is supposed to exist. For surgical safety and simplicity, labyrinthotomy or cochleostomy without posterior tympanotomy has been widely used in re-

Copyright (C) 2022 by Korean Society of Otorhinolaryngology-Head and Neck Surgery.

This is an open-access article distributed under the terms of the Creative Commons Attribution Non-Commercial License (https://creativecommons.org/licenses/by-nc/4.0)

which permits unrestricted non-commercial use, distribution, and reproduction in any medium, provided the original work is properly cited. 

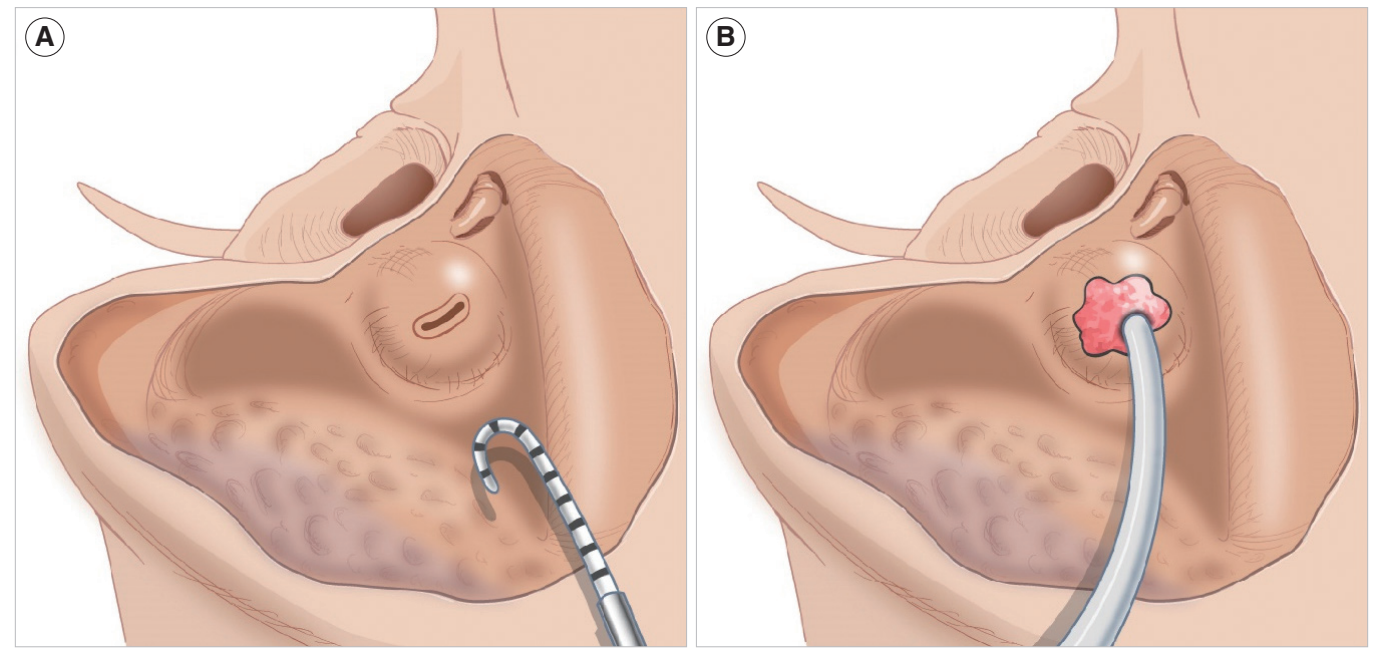

Fig. 1. Schematic drawing of electrode insertion using single-slit labyrinthotomy. (A) Single-slit labyrinthotomy was performed and the electrode was bent before insertion. (B) The labyrinthotomy site was tightly sealed with soft tissue after electrode insertion.

cent studies [7-9]. In 2008, Coelho et al. [10] reported a fluoroscopic technique to reduce the misplacement of electrodes. They reported that electrode misplacement was common and could affect patients' hearing prognosis.

However, there is a paucity of evidence regarding misplaced electrodes in common cavity patients. This may be due to the fact that in these cases, the electrode should be replaced using revision surgery, or it might be ignored if hearing restoration is achieved, regardless of electrode positioning. Thus, the aim of this study was to share our experience with electrode misplacement in patients with common cavity deformity who received cochlear implants.

\section{MATERIALS AND METHODS}

\section{Patients}

The present study was approved by the Institutional Review Board of Severance Hospital (IRB No. 4-2021-0072). Informed consent was waived because this is a retrospective study using

\section{H I G H L I G H T S}

- Misplacement of the electrode tip is not rare in common cavity deformity.

- Regardless of the electrode position, patients showed improved hearing function after cochlear implantation.

- The decision to perform electrode repositioning should be made with careful consideration of possible damage to the cochlear neuroepithelium.

- Electrode repositioning may not be required if there is contact with the inner cavity wall. medical records. From January 2009 to January 2019, 10 cochlear implant surgeries were performed on eight patients with common cavity deformity due to bilateral profound hearing loss as identified in the digital medical database of Severance hospital (Seoul, Korea). The medical records of these patients were retrospectively reviewed. All patients had a temporal magnetic resonance image and temporal computed tomography (CT) conducted before surgery.

\section{Cochlear implant surgery and mapping}

After simple mastoidectomy, the bony wall of the common cavity was exposed. Labyrinthotomy was performed in a single-slit fashion taking care not to insult the facial nerve. Electrodes were manually bent before insertion to prevent misplacement and reduce physical damage to the neuroepithelium. The electrode was inserted through the labyrinthotomy site and tightly sealed with the muscle fascia and fibrin glue to prevent cerebrospinal fluid leakage and ensure the stability of the electrode (Fig. 1).

The $\mathrm{T}$ and $\mathrm{C}$ levels and the dynamic range were measured at the channels located at the basal, mid, and apical sections of the electrode array after placement of the cochlear implants. During the regular mapping, the level of stimulation was adjusted by an experienced audiologist as the patients' response. The channel response was evaluated and turned off if there was no response.

\section{Evaluation of hearing outcome}

Auditory performances were evaluated before surgery, and annually as possible after the switch-on. An aided pure tone threshold test was performed in the sound field. Speech perception ability was assessed using the categories of auditory performance (CAP) score. Ling 6 sound, mono/disyllabic words, and sentence perception tests under auditory-only listening conditions were performed. The tests were performed with samples of words or 
Table 1. Clinical and demographic characteristics of the enrolled patients

\begin{tabular}{lcccccc}
\hline Patient & Operative age $(\mathrm{yr})$ & Sex & Cl side & Electrode & Structural feature & Comorbidity \\
\hline 1 & 9.4 & $\mathrm{M}$ & $\mathrm{Lt}$ & Med-El (ST) & ASCC & None \\
2 & 1.6 & $\mathrm{~F}$ & $\mathrm{Rt}$ & Cochlear (ST) & ASCC, narrowed IAC & None \\
3 & 14.9 & $\mathrm{~F}$ & $\mathrm{Lt}$ & Cochlear (ST) & LSCC & None \\
4 & 6.7 & $\mathrm{M}$ & $\mathrm{Lt}$ & Cochlear (ST) & ASCC, thick bony wall & Intellectual disability \\
5 & 1.8 & $\mathrm{~F}$ & $\mathrm{Rt}$ & Cochlear (ST) & ASCC, narrowed IAC & None \\
6 & 2.4 & $\mathrm{~F}$ & $\mathrm{Lt}$ & Med-El (ST Com) & ASCC, PSCC & None \\
7 & 1.6 & $\mathrm{M}$ & Both & Cochlear (ST) & Rt ASCC, Lt ASCC, PSCC, LSCC & None \\
8 & 0.7 & M & Both & Cochlear (ST) & Rt severely narrowed IAC & None \\
\end{tabular}

Cl, cochlear implant; LT, left; ST, straight; ASCC, anterior semicircular canal; Rt, right; IAC, internal auditory canal; LSCC, lateral semicircular canal; Com, compressed; PSCC, posterior semicircular canal.
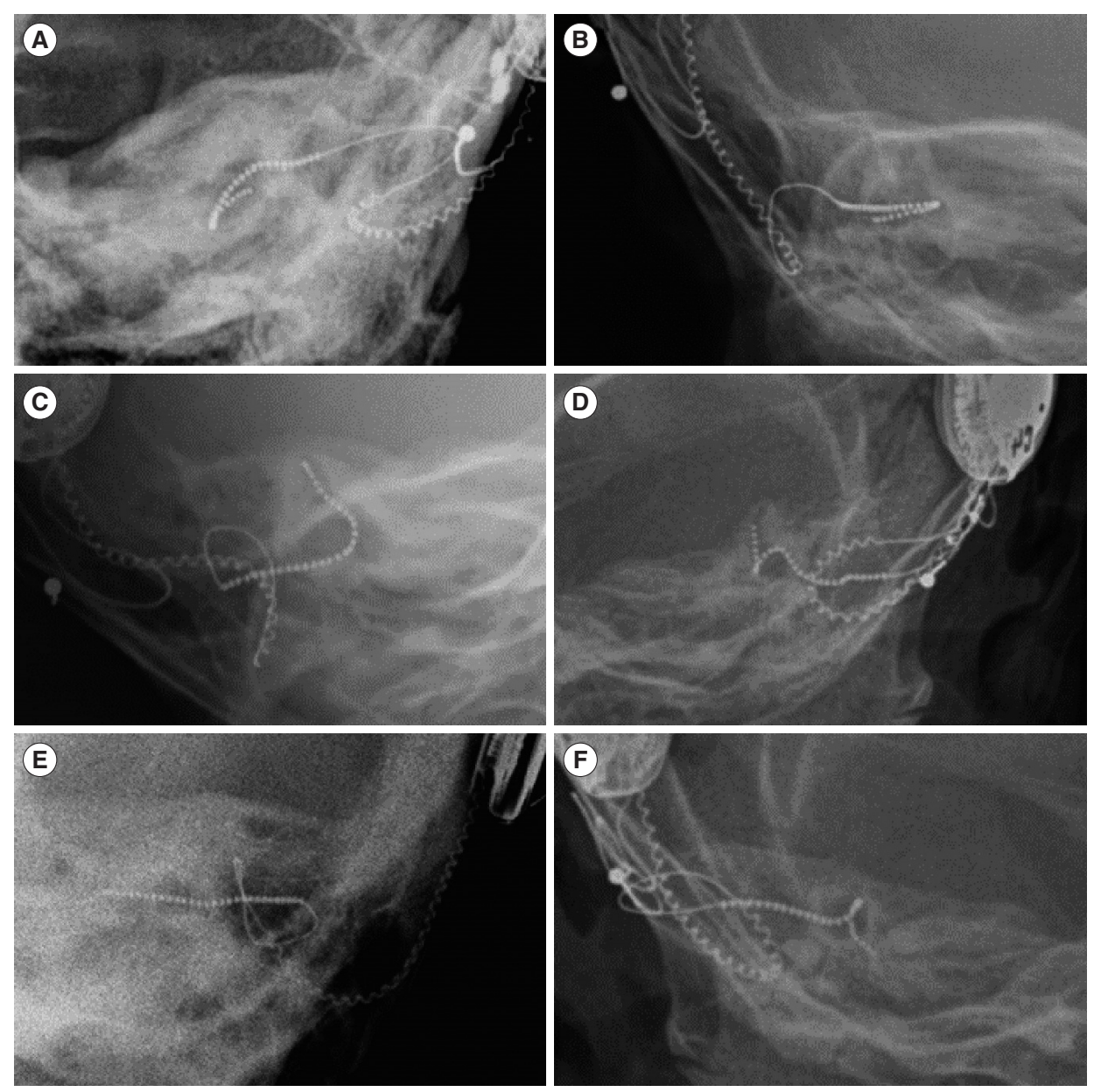

Fig. 2. Transorbital ocular view of the electrodes. (A, B) The properly placed electrodes show a U-shape and the tips are pointing in the lateral direction. (C, D) Misplaced electrodes in the anterior semicircular canal and (E, F) the internal auditory canal. (E) The electrode in patient 3 did not make contact with the inner wall of the cavity. Of note, this is the only electrode that did not bend along the inner wall.

sentences used in everyday life in a noiseless sound-field environment. The tests were scored as the percent of words that were correctly repeated. The most recent results from the annual hearing assessments have been described in the present study.

\section{Evaluation of electrode position}

A trans-orbital ocular view image was obtained after surgery to confirm the position of the electrode. Electrode misplacement was identified if it did not show a U-shape because the tip of the electrode was not in the lateral position. 

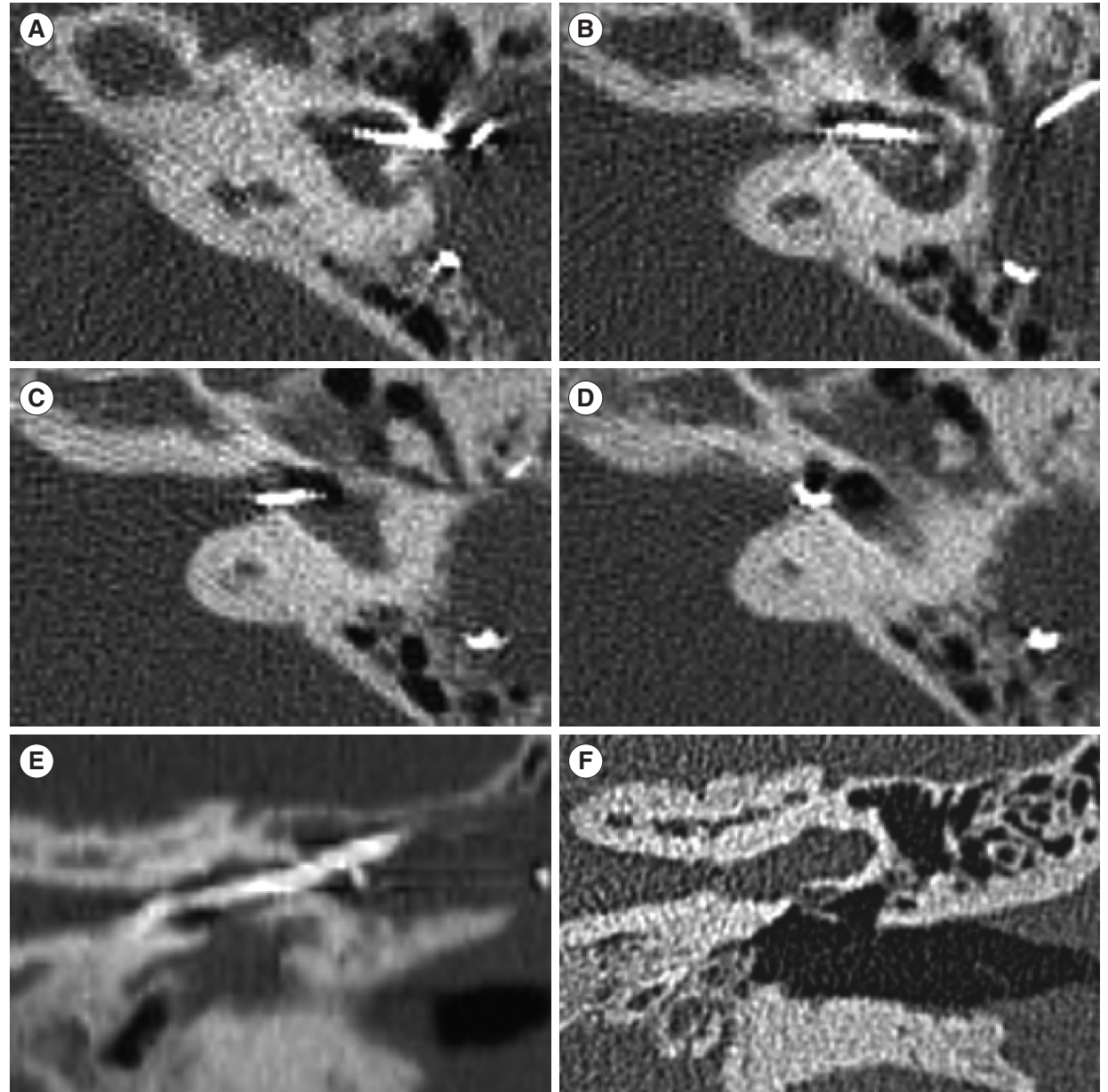

Fig. 3. Computed tomography images of patient 3. (A-D) Axial images confirmed that the electrode did not come into contact with the inner wall of the cavity. (E) Coronal image of the straightly positioned electrode. (F) Coronal image of the same plane before surgery.

\section{RESULTS}

Clinical and demographic details of the enrolled patients

All the enrolled patients had congenital bilateral profound hearing loss. The age at which cochlear implant operations were conducted ranged from 0.7 to 14.9 years (Table 1). Four patients were male and two patients received bilateral simultaneous cochlear implants. A straight-type electrode was used for all patients, and a compressed electrode was used for patient 6 . Two Med-El (Med-El, Innsbruck, Austria) and eight Cochlear devices were used, respectively. All the implanted ears had a discernible cochleovestibular nerve (CN VIII), but none of them had a discriminable cochlear nerve from CN VIII. The structural features of the common cavity were very different for each patient. The anterior semicircular canal was identifiable in the majority of cases (7 out of 10 ears). Patient 4 had concurrent intellectual disability associated with Emanuel syndrome.

\section{Electrode misplacement}

Four of the 10 implants were identified as misplaced on postoperative transorbital ocular view images (Fig. 2). Two electrodes were placed in the superior semicircular canal and the other two electrodes were in contact with the fundus of the IAC. All implanted electrodes, with the exception of those in patient 3 , were bent inside the cavity and appeared to be in contact with the inner wall of the cavity. A postoperative high-resolution CT scan of patient 3 indicated that the electrode had no contact with the inner wall of the cavity, and the tip of the electrode was kinked in front of the IAC fundus (Fig. 3).

\section{Perioperative complications}

There were no severe cerebrospinal fluid gushers after labyrinthotomy in any patient during surgery. Patient 4 , however, experienced failed labyrinthotomy after the first surgery because he had a very thick cavity wall and a poorly pneumatized mastoid cavity, which is an uncommon finding considering his age. No patients experienced meningitis during the follow-up period. 
Table 2. Hearing outcomes of the enrolled patients

\begin{tabular}{|c|c|c|c|c|c|c|c|c|c|}
\hline Patient & Side & Active channel & Latest follow-up period (yr) & Threshold (dB) & CAP & Ling 6 & MSW (\%) & DSW (\%) & Sentence (\%) \\
\hline 1 & $\mathrm{Lt}$ & $10 / 12$ & 1.8 & 37.5 & $\mathrm{NC}$ & $\mathrm{NC}$ & NC & $\mathrm{NC}$ & $\mathrm{NC}$ \\
\hline 2 & Rt & $19 / 22$ & 4.2 & 32.5 & 4 & 6 & 40 & 50 & 0 \\
\hline 3 & $\mathrm{Lt}^{\mathrm{a})}$ & $6 / 22$ & 2.0 & 35.0 & 3 & 1 & 0 & 0 & 0 \\
\hline 4 & $\mathrm{Lt}$ & 20/22 & $\mathrm{NC}$ & $\mathrm{NC}$ & NC & $\mathrm{NC}$ & $\mathrm{NC}$ & $\mathrm{NC}$ & $\mathrm{NC}$ \\
\hline 5 & Rt & 22/22 & 4.8 & 32.5 & 4 & 4 & 0 & 0 & 0 \\
\hline 6 & $\mathrm{Lt}$ & $10 / 12$ & 5.0 & 37.5 & $\mathrm{NC}$ & $\mathrm{NC}$ & $\mathrm{NC}$ & $\mathrm{NC}$ & $\mathrm{NC}$ \\
\hline \multirow[t]{2}{*}{7} & $\mathrm{R}^{\mathrm{b})}$ & 22/22 & 2.0 & 37.5 & 5 & 6 & 10 & 10 & 0 \\
\hline & $\mathrm{Lt}$ & 22/22 & & 30.0 & & & & & \\
\hline \multirow[t]{2}{*}{8} & $\mathrm{Rt}^{\mathrm{a})}$ & 22/22 & 1.5 & $\mathrm{NC}$ & 4 & 3 & 0 & 0 & 0 \\
\hline & $L t^{b)}$ & $14 / 22$ & & $\mathrm{NC}$ & & & & & \\
\hline
\end{tabular}

CAP, categories of auditory performance; MSW, monosyllabic word discrimination; DSW, disyllabic word discrimination; Lt, left; NC, not conducted; Rt, right.

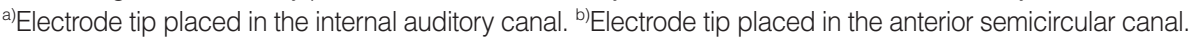
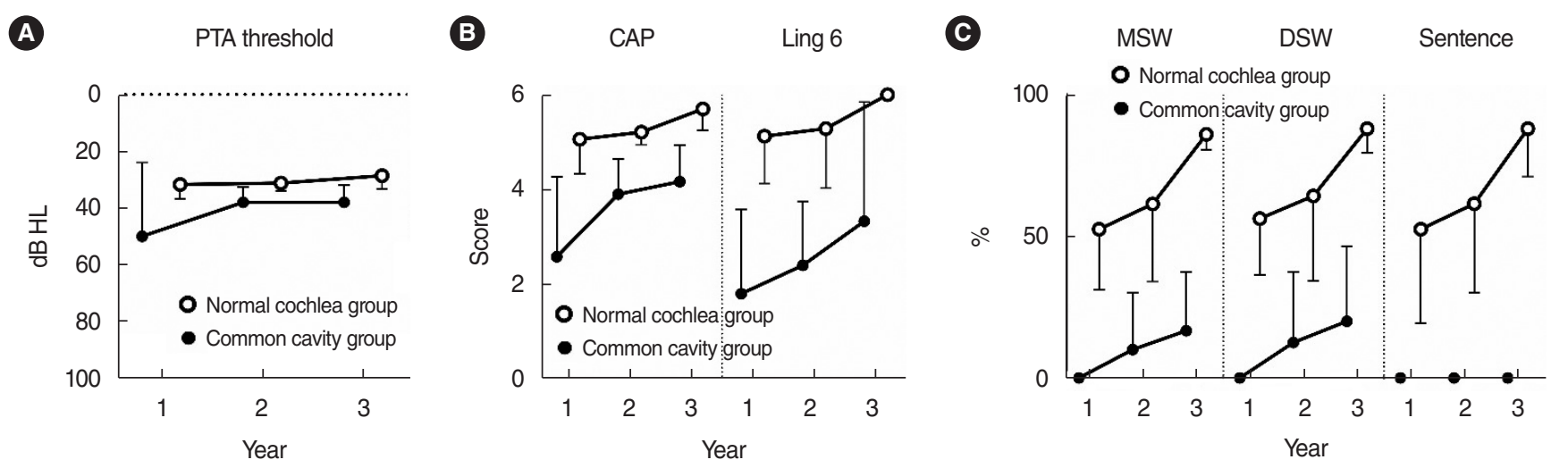

Fig. 4. Hearing outcomes of the common cavity group and normal cochlea group. values are presented as mean and standard deviation. (A) Pure tone audiometry (PTA) threshold. (B) Categories of auditory performance (CAP) score and Ling 6 score. (C) Speech intelligibility test results. A statistical analysis was not conducted due to the small number of patients in the common cavity group. MSW, monosyllabic word discrimination; DSW, disyllabic word discrimination.

Patient 2 developed skin necrosis where the device was placed 5 years after the operation. Wound debridement followed by a local skin flap graft was conducted; however, skin necrosis recurred. The skin necrosis was finally resolved after re-implantation of a new device in revision surgery.

\section{Hearing outcomes}

The patients who received cochlear implants and underwent an aided pure tone threshold test in the sound field showed a hearing threshold of approximately $35 \mathrm{~dB}$ (Table 2). However, their hearing performance was not encouraging; in particular, they showed worse performance in speech intelligibility. None of the five patients who underwent the language assessment could distinguish a sentence. Meanwhile, the CAP score and Ling 6 test showed improvement after the cochlear implant was placed. The proportion of active electrodes was $64 \%-100 \%$, with the exception of a markedly lower level in patient $3(27 \%)$.

We reviewed the hearing outcomes of patients who received cochlear implants with a normal cochlea on temporal bone imaging as a control group from our institutional database. Since the mean operative age of the enrolled common cavity deformity patients was 4.89 years, eight of the normal-cochlea patients with an operative age of 5 years were enrolled as the control group. The common cavity group showed limited hearing function in all categories compared to the control group, especially in speech intelligibility tests (Fig. 4).

\section{DISCUSSION}

The present study enrolled eight patients with common cavity deformity who received cochlear implants. Among them, four electrodes were identified as having been misplaced, as their tips were not appropriately located in the cavity. Regardless of the electrode position, all patients gained auditory perception and had improved hearing function after cochlear implantation. Sixty-four percent to $100 \%$ of the electrodes were responsive, except in one patient in whom the implant was ascertained to have no contact to the inner wall of the cavity and had only $27 \%$ of electrodes that were active. 
The hearing outcomes for cochlear implants in common cavity cases are generally poor, with the worst outcomes among those with cochlear deformations after long-term follow-up [3,11-13]. Our study also identified a poor prognosis, especially in language performance. Nevertheless, there are obvious advantages of cochlear implantation that justify the risks of the surgery $[14,15]$. Perilymph gushers have been commonly reported in previous studies; however, none of the enrolled patients in this study had severe gushers that required extensive sealing $[9,11,16,17]$. Recurrent meningitis is also supposed to be a critical complication after cochlear implantation in patients with common cavity deformity [18]. In the present study, however, no patients were reported to have recurrent meningitis. Thorough sealing of the labyrinthotomy site is important for controlling these perioperative complications. The labyrinthotomy procedure is easy and safe in the majority of patients; however, if it is expected to be challenging, as in patient 4 in the present study, it should be carefully planned and executed by an experienced surgeon.

U-shaped electrodes in the cavity are considered the best position to maximize stimulation of the cochlear neuroepithelium, which is expected to be located in the inner wall of the cavity near the IAC fundus. Thus, many suggested surgical approaches seek to obtain a U-shaped electrode position. Beltrame et al. $[5,19]$ suggested a double posterior labyrinthotomy technique that ensures that the electrode is bent along the inner wall of the cavity by pushing and pulling the electrode tip. More recently in 2018, Wei et al. [20] reported a custom-made U-shaped electrode placed through a single-slit labyrinthotomy. In the current study, a misplaced electrode was defined as not being Ushaped. Among the 10 cases, $40 \%(n=4)$ showed misplacement; this proportion is similar to that (two of four implants) reported by Coelho et al. [10]. Notably, the patients with misplaced electrodes also showed improved hearing function after implant surgery, with CAP scores of 3-5. The poor hearing outcome of patient 3 most likely primarily resulted from the old operative age (14.9 years old) and short follow-up period ( 2 years). However, due to the limited number of active electrodes, it is possible that poor contact of the electrode with the inner cavity wall might have contributed to the poor hearing outcome. This implies that regardless of the tip position, contact with the inner cavity wall may sufficiently stimulate the cochlear neuroepithelium. Since electrode insertion can physically damage the fragile neuroepithelium, electrode repositioning is not recommended when the electrode exhibits bending along the inner wall.

There are some limitations in the present study. First, we did not compare cochlear implant performance before and after the repositioning of misplaced electrodes. Thus, it is possible that hearing performance may improve further if the electrode is repositioned in a U-shape. Second, a more robust analysis could have been performed if all enrolled patients had undergone all the hearing tests. Lastly, language evaluation was conducted using bilateral cochlear implants in patients 7 and 8 . Poor functional- ity of one device could have been concealed by the contralateral well-functioning device. A prospective study could resolve these issues in the future.

The current study found that in patients with common cavity deformity, even misplaced electrodes could perform well if they were in contact with the inner wall of the cavity. The decision regarding electrode repositioning should be made carefully, considering the potential physical damage to the cochlear neuroepithelium. These findings can inform surgeons about predicted complications associated with cochlear implantation for patients with common cavity deformity and provide insights as to the required revisions to surgery.

\section{CONFLICT OF INTEREST}

No potential conflict of interest relevant to this article was reported.

\section{ACKNOWLEDGMENTS}

This research was supported by the Basic Science Research Program through the National Research Foundation of Korea (NRF) funded by the Ministry of Education (NRF-2020R1I1A1A01067241 to SHB).

The authors thank Medical Illustration \& Design, part of the Medical Research Support Services of Yonsei University College of Medicine, for all artistic support related to this work.

The authors would like to express special thanks to audiologist JH Heo.

\section{ORCID}

Seong Hoon Bae https://orcid.org/0000-0001-9243-9392

Jihoon Choi https://orcid.org/0000-0002-0465-3098

Jae Young Choi https://orcid.org/0000-0001-9493-3458

\section{AUTHOR CONTRIBUTIONS}

Conceptualization: JYC. Data curation: JC. Formal analysis: SHB. Funding acquisition: SHB. Methodology: SHB. Project administration: JC. Visualization: SHB. Writing-original draft: JYC, SHB.Writing-review \& editing: JYC, SHB.

\section{REFERENCES}

1. Brotto D, Avato I, Lovo E, Muraro E, Bovo R, Trevisi P, et al. Epidemiologic, imaging, audiologic, clinical, surgical, and prognostic issues 
in common cavity deformity: a narrative review. JAMA Otolaryngol Head Neck Surg. 2019 Jan;145(1):72-8.

2. Shi Y, Li Y, Gong Y, Chen B, Chen J. Cochlear implants for patients with inner ear malformation: experience in a cohort of 877 surgeries. Clin Otolaryngol. 2019 Jul;44(4):702-6.

3. Ozkan HB, Cicek Cinar B, Yucel E, Sennaroglu G, Sennaroglu L. Audiological performance in children with inner ear malformations before and after cochlear implantation: a cohort study of 274 patients. Clin Otolaryngol. 2021 Jan;46(1):154-60.

4. Pradhananga RB, Thomas JK, Natarajan K, Kameswaran M. Long term outcome of cochlear implantation in five children with common cavity deformity. Int J Pediatr Otorhinolaryngol. 2015 May; 79(5):685-9.

5. Beltrame MA, Birman CS, Cervera Escario J, Kassouma J, Manolidis $\mathrm{S}$, Pringle MB, et al. Common cavity and custom-made electrodes: speech perception and audiological performance of children with common cavity implanted with a custom-made MED-EL electrode. Int J Pediatr Otorhinolaryngol. 2013 Aug;77(8):1237-43.

6. Miyamoto RT, Robbins AJ, Myres WA, Pope ML. Cochlear implantation in the Mondini inner ear malformation. Am J Otol. $1986 \mathrm{Jul}$; 7(4):258-61.

7. Bako P, Nemeth A, Molnar K, Toth T, Harmat K, Rath G, et al. Cochlear implantation in patients afflicted with inner ear malformations: the Pecs experience. Hearing Balance Commun. 2018;16(4): 232-7.

8. Sennaroglu L. Cochlear implantation in inner ear malformations: a review article. Cochlear Implants Int. 2010 Mar;11(1):4-41.

9. Xia J, Wang W, Zhang D. Cochlear implantation in 21 patients with common cavity malformation. Acta Otolaryngol. 2015 May;135(5): 459-65.

10. Coelho DH, Waltzman SB, Roland JT Jr. Implanting common cavity malformations using intraoperative fluoroscopy. Otol Neurotol. 2008 Oct;29(7):914-9.
11. Ahn JH, Lim HW, Lee KS. Hearing improvement after cochlear implantation in common cavity malformed cochleae: long-term followup results. Acta Otolaryngol. 2011 Sep;131(9):908-13.

12. Catli T, Uckan B, Olgun L. Speech and language development after cochlear implantation in children with bony labyrinth malformations: long-term results. Eur Arch Otorhinolaryngol. 2015 Nov;272(11): 3131-6.

13. Daneshi A, Farhadi M,Ajalloueyan M, Rajati M, Hashemi SB, Ghasemi MM, et al. Cochlear implantation in children with inner ear malformation: a multicenter study on auditory performance and speech production outcomes. Int J Pediatr Otorhinolaryngol. 2020 May; 132:109901.

14. Irvine DR. Auditory perceptual learning and changes in the conceptualization of auditory cortex. Hear Res. 2018 Sep;366:3-16.

15. Zhang L, Qiu J, Qin F, Zhong M, Shah G. Cochlear implantation outcomes in children with common cavity deformity: a retrospective study. J Otol. 2017 Sep;12(3):138-42.

16. Dettman S, Sadeghi-Barzalighi A, Ambett R, Dowell R, Trotter M, Briggs R. Cochlear implants in forty-eight children with cochlear and/or vestibular abnormality. Audiol Neurootol. 2011;16(4):222-32.

17. Loundon N, Rouillon I, Munier N, Marlin S, Roger G, Garabedian EN. Cochlear implantation in children with internal ear malformations. Otol Neurotol. 2005 Jul;26(4):668-73.

18. Bille J, Fink-Jensen V, Ovesen T. Outcome of cochlear implantation in children with cochlear malformations. Eur Arch Otorhinolaryngol. 2015 Mar;272(3):583-9.

19. Beltrame MA, Bonfioli F, Frau GN. Cochlear implant in inner ear malformation: double posterior labyrinthotomy approach to common cavity. Adv Otorhinolaryngol. 2000;57:113-9.

20. Wei X, LiY, Fu QJ, Gong Y, Chen B, Chen J, et al. Slotted labyrinthotomy approach with customized electrode for patients with common cavity deformity. Laryngoscope. 2018 Feb;128(2):468-72. 